\title{
Diferencias en la percepción de actividad física en escolares de sexto de primaria Differences in the perception of physical activity in sixth-grade primary school students
}

Raúl Jiménez Boraita, Josep María Dalmau Torres, Esther Gargallo Ibort, Daniel Arriscado Alsina Universidad de La Rioja (España)

\begin{abstract}
Resumen. El objetivo del estudio fue analizar la autopercepción del nivel de actividad física de los escolares de sexto curso de Educación Primaria y contrastarla con la percepción de sus maestros de Educación Física. Se administró el Physical Activity Questionnaire for Older Children a una muestra representativa de 329 escolares de sexto curso (11-12 años) de los 31 centros educativos de Logroño (La Rioja). Igualmente, un maestro de Educación Física de cada centro completó el cuestionario por duplicado, reportando su percepción para el caso de los chicos y de las chicas. También se preguntó por el tiempo frente a la pantalla. Los niveles de Actividad Física fueron estadísticamente superiores en los chicos, especialmente, en las clases de Educación Física, los recreos y los fines de semana. Estas diferencias fueron percibidas por los maestros en los dos últimos casos. Al contrastar las respuestas de los chicos y sus profesores, estos sobreestimaron la Actividad Física realizada por sus alumnos antes y después de comer, subestimando la práctica realizada los fines de semana. En el caso de las chicas, también subestimaron su actividad durante los fines de semana, así como la del global de la semana. Por último, respecto al tiempo de pantalla, no hubo diferencias entre sexos, pero en ambos casos los profesores sobreestimaron el tiempo reportado por su alumnado. Dadas las diferencias encontradas, es preciso analizar los hábitos de práctica de nuestro alumnado, comprenderlos y, de este modo, intervenir de forma eficaz, especialmente, desde las escuelas como elementos clave de promoción de la salud.
\end{abstract}

Palabras claves: actividad física, percepción, niño, escuela, educación física.

Abstract. The objective of the present study was to analyse perceptions of personal engagement in physical activity in sixth-grade primary school students, and to compare them with the perception of their physical education (PE) teachers. A representative sample of 329 sixth graders (aged 11-12 years old) from 31 educational centres in Logroño (La Rioja) completed the Physical Activity Questionnaire for Older Children. In addition, a PE teacher from each educational centre completed the same questionnaire, self-reporting their perceptions of physical activity with regards to the boys and girls attending their centre. Screen-time was also reported. Boys engaged in significantly more physical activity than girls, specifically during PE classes, during break-times, and at weekends. Teachers corroborated these reports in the case of the latter two contexts. Differences existed between the perceptions reported by the teachers and male students. On one hand the teachers overestimated physical activity engagement of their male students before and after eating, on the other they underestimated their physical activity at weekends. Teacher reports also differed from those of female students, with teachers underestimating their physical activity both at weekends and overall throughout the week. Finally, with respect to screen-time, no differences were reported between genders, but teachers overestimated this variable for both male and female students. Given the differences identified, it is important to analyse the habits of young scholars, understand them and, in this way, design efficacious interventions, particularly utilising schools as key providers in the promotion of health.

Key words: physical activity, perception, child, school, physical education.

\section{Introducción}

La Organización Mundial de la Salud (OMS) estimó en 2016 que 340 millones de niños y adolescentes, entre 5 y 19 años, padecen sobrepeso u obesidad (OMS, 2017). Estos datos denotan que nos encontramos ante un problema de salud pública, al que la OMS ha denominado la «Epidemia del Siglo XXI». En España, el estudio ALADINO, realizado en el año 2015 en la población infantil entre 6 y 9 años, señaló que el porcentaje de prevalencia de obesidad en este rango de edad se encontraba en un $18,1 \%$ y en un $23,2 \%$ el de sobrepeso.

Esta situación derivada fundamentalmente de los actuales hábitos de alimentación y práctica de actividad física (AF), requiere un tratamiento interdisciplinar de colaboración entre diferentes ámbitos (Williams, Mesidor, Winters, Dubbert, Wyatt, 2015). La AF realizada regularmente reporta beneficios sobre la salud física, relacionándose además con diferentes aspectos de la salud mental y social de los jóvenes como el autoconcepto, la satisfacción de vida, la calidad de las relaciones o el menor consumo de sustancias nocivas (Bymer y Davids, 2016 \& Iannotti et al., 2009). Sin embargo,

Fecha recepción: 18-02-19. Fecha de aceptación: 30-05-19 Raúl Jiménez Boraita rauljbcity@gmail.com estudios como el de Lavielle, Pineda, Jáuregui, Castillo (2014) determinaron que la práctica de AF es menos frecuente de lo deseable en la población adolescente e infantil, convirtiendo esta realidad en elemento clave a revertir en aras de la salud pública.

En la actualidad, el ocio tiene un papel muy relevante en la actividad diaria de la población, y es en este espacio, donde se brinda una gran oportunidad de dedicación de un mayor tiempo para la práctica. Este uso del ocio saludable se constata significativo si tenemos en cuenta estudios como el de Ramos, Rivera, Jiménez, y Moreno (2012) donde se halló que el 45,1\% de chicas y el 30\% de chicos no realizan 60 minutos de AF diaria, tiempo mínimo recomendado según la OMS (2010).

Centrándonos en el entorno escolar y considerando los centros educativos como entornos de promoción de la salud, resalta la potencialidad que estos poseen de influir en las conductas del alumnado a la hora de adoptar un estilo de vida más saludable (Arriscado, Muros, Zabala y Dalmau, 2015). De manera más concreta, el área de educación física ( EF) debe ser considerada como un referente en la promoción de hábitos saludables, tratando de promover hábitos de AF durante el tiempo de ocio con el fin de aumentar la práctica física diaria del alumnado (Delgado y Tercedor, 2002). En este sentido, es importante mencionar la valoración positiva que tiene la materia de EF tanto para las familias (Gil, Perona, 
Prieto y Sáez, 2017) como para los alumnos (Oyarzún, Wydra, Hernández y Salazar, 2017), hecho que sitúa a esta materia en una posición privilegiada para la promoción de hábitos saludables entre los estudiantes.

El objetivo de este estudio, se centra en el análisis de la autopercepción de AF de los escolares de sexto curso de Educación Primaria y el contraste con la percepción de sus maestros de EF. Los resultados obtenidos aportan información de utilidad para conocer la realidad de la práctica de AF por parte de los estudiantes para que, desde el entorno escolar, se puedan realizar programas de prevención, formación y promoción de este tipo de actividades con el fin de fomentar hábitos saludables en el alumnado.

\section{Método}

\section{Muestra}

Se diseñó un estudio descriptivo transversal que atendiera, por un lado, las opiniones de los escolares en sexto curso de Educación Primaria y, por el otro, las de sus profesores. De este modo, la investigación contó con dos poblaciones de estudio.

En cuanto a los escolares, se realizó un muestreo aleatorio simple entre todos los centros educativos públicos y concertados de la ciudad, lo que supuso una muestra representativa de 329 alumnos (11,7 $\pm 0,4$ años ). Se eliminó del análisis a once sujetos que no pudieron realizar práctica física con normalidad o que no completaron los cuestionarios correctamente, quedando 318 escolares. Además, se solicitó el consentimiento informado de los progenitores o tutores.

Por otro lado, se solicitó la colaboración de los maestros de EF de sexto curso de cada uno de los centros en los que se realizó el estudio. La muestra obtenida fue de 31 maestros, coincidiendo con el número de centros existentes en la localidad de Logroño. Sólo se solicitó la participación de uno de los docentes de EF en el caso de los centros donde coexistían docentes con estas mismas funciones en sexto curso de Educación Primaria.

Todos los participantes tomaron parte de manera voluntaria, respetando el acuerdo sobre ética de investigación de Helsinki. El proyecto de esta investigación obtuvo el consentimiento del Comité Ético de Investigación Clínica de La Rioja.

\section{Instrumentos}

Se añadió un ítem inicial en el cuestionario donde los propios participantes informaron de su género. Con el objetivo de determinar el nivel de AF del alumnado, éstos completaron el cuestionario Physical Activity Questionnaire for Older Children (PAQ-C), validado y adaptado al idioma español (Martínez et al., 2009). El cuestionario pretende valorar el nivel de intensidad (moderada a vigorosa) de AF practicada en los últimos siete días. Se compone de nueve preguntas que tratan sobre el tipo de actividad y la frecuencia con la que realizaron $\mathrm{AF}$ en diferentes momentos del día durante la última semana. De las respuestas se obtiene una puntuación del 1 al 5, siendo mayor cuanto más activo es el niño. Además, se preguntó al alumnado por las horas al día dedicadas a la visualización de la televisión, el ordenador y el uso de videojuegos. Todos los cuestionarios destinados a los estu- diantes fueron administrados de forma guiada por un investigador entrenado y bajo la supervisión de tres colaboradores que aseguraban la comprensión por parte de los participantes.

Por otro lado, el maestro de EF de sexto curso de cada escuela, contestó a las preguntas sobre hábitos de AF por duplicado, una vez refiriéndose a los chicos y otra a las chicas, reflejando así su percepción de la AF que unos y otras realizan.

\section{Análisis Estadístico}

Las variables cuantitativas se presentan con la media y su desviación típica. La normalidad de los datos fue analizada usando el test de Kolmogorov-Smirnov. Una vez comprobada la normalidad, la comparación de las medias entre los diferentes grupos se realizó a través de la prueba $\mathrm{T}$, en el caso de las variables con distribución normal, y de la prueba U de Mann-Whitney, para las variables con distribución no normal.

Los datos fueron analizados con el programa estadístico IBM-SPSS versión 25 para Windows. El nivel de significación se estableció en 0,05.

\section{Resultados}

La tabla 1 muestra las respuestas según el género de los escolares a las cuestiones relacionadas con los hábitos y niveles de AF. De este modo, se puede observar que los chicos reportaron niveles significativamente mayores que las chicas en todas las cuestiones referentes a la práctica de AF, excepto a la hora de la comida y entre las 18 y las 22 horas de la tarde. Además, tampoco se hallaron diferencias significativas en relación a las horas de televisión, ordenador o utilización de videojuegos.

La tabla 2 ilustra las diferencias entre la percepción del alumnado de género masculino y la de los maestros para este alumnado. Las diferencias significativas encontradas, denotan una sobrestimación por parte de los maestros sobre los niveles de AF del alumnado masculino en los momentos previos y posteriores a la hora de la comida, durante los días de la semana y en el uso diario de la televisión, ordenador o videojuegos. Además, también se encontraron diferencias significativas en la AF realizada durante el fin de semana, donde la percepción de los maestros subestimó la práctica del alumnado.

En cuanto a las diferencias entre el alumnado de género femenino y la percepción de los maestros, en la tabla 3 se puede observar que se hallaron diferencias significativas $(p<0,001)$ en la AF realizada durante el fin de semana y en el global de la semana, donde los valores de percepción de los maestros eran inferiores a los del alumnado. Además, también se encontró una diferencia significativa $(p<.001)$ en el uso diario de televisión, ordenador y videojuegos, donde los maestros sobrestimaron la utilización de estos por parte del alumnado femenino.

Por último, si atendemos a la percepción de los maestros sobre la AF para cada uno de los géneros, en la tabla 4 se puede apreciar que se hallaron valores superiores en todos los aspectos relacionados con la AF por parte de los chicos, además, estas diferencias fueron significativas en todas las 
Tabla 1.

\begin{tabular}{|c|c|c|}
\hline Actividad física en la última semana & $\begin{array}{l}\text { Chicos } \\
(\mathrm{n}=160)\end{array}$ & $\begin{array}{l}\text { Chicas } \\
(\mathrm{n}=158)\end{array}$ \\
\hline Actividades y deportes realizados & $1,78 \pm 0,37 * * *$ & $1,65 \pm 0,34$ \\
\hline En Educación Física & $4,24 \pm 0,78^{* * *}$ & $3,91 \pm 0,91$ \\
\hline En el recreo & $4,17 \pm 0,91 * * *$ & $3,25 \pm 1,13$ \\
\hline Justo antes/después de comer & $2,14 \pm 1,21$ & $1,89 \pm 1,15$ \\
\hline Hasta las 18 horas de la tarde & $3,18 \pm 1,26^{*}$ & $2,84 \pm 1,13$ \\
\hline De 18 a 22 horas de la tarde & $2,99 \pm 1,18$ & $2,95 \pm 1,12$ \\
\hline El fin de semana & $3,25 \pm 1,06^{* *}$ & $2,92 \pm 1,00$ \\
\hline Global de la semana & $3,34 \pm 0,97 * * *$ & $2,89 \pm 1,00$ \\
\hline Los diferentes días de la semana & $3,30 \pm 0,69 * * *$ & $2,97 \pm 0,65$ \\
\hline PUNTUACIÓN TOTAL & $3,16 \pm 0,55^{* * * *}$ & $2,81 \pm 0,52$ \\
\hline $\begin{array}{l}\text { Horas diarias de televisión, ordenador y } \\
\text { videojuegos }\end{array}$ & $1,6 \pm 0,93$ & $1,55 \pm 0,81$ \\
\hline
\end{tabular}

Tabla 2 .

\begin{tabular}{|c|c|c|}
\hline Actividad física en la última semana & Chicos $(n=160)$ & Percepción maestros $(\mathrm{n}=31)$ \\
\hline En Educación Física & $4,24 \pm 0,78$ & $4,40 \pm 0,50$ \\
\hline En el recreo & $4,17 \pm 0,91$ & $4,27 \pm 0,45$ \\
\hline Justo antes/después de comer & $2,14 \pm 1,21 * *$ & $2,90 \pm 1,42$ \\
\hline Hasta las 18 horas de la tarde & $3,18 \pm 1,26$ & $3,33 \pm 0,88$ \\
\hline De 18 a 22 horas de la tarde & $2,99 \pm 1,18$ & $3,03 \pm 0,67$ \\
\hline El fin de semana & $3,25 \pm 1,06 *$ & $2,80 \pm 0,93$ \\
\hline Global de la semana & $3,34 \pm 0,97$ & $3,23 \pm 1,01$ \\
\hline Los diferentes días de la semana & $3,30 \pm 0,69 * * *$ & $3,83 \pm 0,83$ \\
\hline $\begin{array}{l}\text { Horas diarias de televisión, ordenador y } \\
\text { videojuegos }\end{array}$ & $1,6 \pm 0,93^{* *}$ & $2,03 \pm 0,72$ \\
\hline
\end{tabular}

Tabla 3.

Autopercepción y percepción de los maestros de EF del nivel de AF en las chicas

\begin{tabular}{lll} 
Autopercepción y percepción de los maestros de EF del nivel de AF en las chicas \\
\hline Actividad física en ñla última semana & Chicas (n=158) & Percepción maestros (n=31)
\end{tabular}

En el recreo

Justo antes/después de comer

Hasta las 18 horas de la tarde

De 18 a 22 horas de la tarde

El fin de semana

Los diferentes días de la semana

Los diferentes días de la semana

Horas diarias

$1,55 \pm 0,81^{* * *}$

Diferencias significativas entre géneros: $p<.001^{* * *}$.

Tabla 4.

Percepción de los maestros de EF del nivel de AF según el género

\begin{tabular}{lcc}
\multicolumn{3}{l}{ Percepción de los maestros de $E F$ del nivel de AF según el género } \\
\hline Actividad física en la última semana & $\begin{array}{c}\text { Percepción maestros } \\
\text { chicos }(\mathrm{n}=31)\end{array}$ & $\begin{array}{c}\text { Percepción maestros } \\
\text { chicas (n=31) }\end{array}$ \\
\hline En Educación Física & $4,40 \pm 0,50$ & $4,26 \pm 0,45$ \\
En el recreo & $4,27 \pm 0,45^{* * *}$ & $2,90 \pm 0,79$ \\
Justo antes/después de comer & $2,90 \pm 1,42^{* *}$ & $2,00 \pm 0,97$ \\
Hasta las 18 horas de la tarde & $3,33 \pm 0,88^{*}$ & $2,84 \pm 0,58$ \\
De 18 a 22 horas de la tarde & $3,03 \pm 0,67$ & $2,77 \pm 0,72$ \\
El fin de semana & $2,80 \pm 0,93^{*}$ & $2,29 \pm 0,59$ \\
Global de la semana & $3,23 \pm 1,01^{* * *}$ & $2,32 \pm 0,65$ \\
Los diferentes días de la semana & $3,83 \pm 0,83^{* * *}$ & $3,03 \pm 0,55$ \\
\hline Horas diarias de televisión, ordenador y & $2,03 \pm 0,72$ & $2,10 \pm 0,91$ \\
videojuegos &
\end{tabular}

variables propuestas a excepción de las clases de EF y el periodo de tiempo entre las 18 y 22 horas de la tarde, donde no se hallaron diferencias. Solamente se percibe mayor dedicación de las chicas hacia las actividades de pantalla, a pesar de que no existen diferencias significativas

\section{Discusión}

En relación a los resultados obtenidos en el presente estudio, atendiendo a las diferencias encontradas en la realización de AF en función del género, nuestro estudio coincide con otras investigaciones como las de Pinel et al. (2017) o Kalman et al. (2015), donde se hallaron mayores niveles de AF en niños que en niñas a lo largo todas las franjas de edad entre los 11 a los 15 años en diferentes países europeos.

En lo relativo a la AF realizada durante los diferentes momentos del día, cabe destacar que no se encontraron diferencias entre chicos y chicas antes/después de comer, coincidiendo con los estudios de Manchola, Bagur y Girabent (2017), donde la realización de AF durante este tramo del día era muy baja tanto en chicos como en chicas. Atendiendo a la franja horaria de 18 a 22 horas, cabe destacar que no se encontraron diferencias, posiblemente porque coincide con el horario para la práctica de actividades organizadas. En este sentido, autores como Ara et al. (2006) y Reverter, Plaza, Jové y Hernández (2014) manifestaron que la realización de AF extraescolar es muy importante para la salud, ya que complementa el nivel de AF realizado por el alumnado durante las clases de EF, que en muchos casos es insuficiente.

Además, si hacemos referencia a las diferencias encontradas de AF durante el fin de semana, podemos observar como los chicos tienen un mayor nivel de AF durante este periodo de la semana. Estos resultados concurren con los de Beltrán et al. (2017), donde se analizó la AF realizada en los diferentes momentos del fin de semana y encontraron concretamente que los chicos hacían más AF que las chicas en el tramo de las 16:00 y 18:30. Cabrero et al. (2015) concluyeron en su estudio que los chicos destinaban más tiempo a AF dirigidas a la competición que las chicas, lo que podría explicar esta diferencia durante la realización de AF en el fin de semana, ya que es cuando coincide con el espacio dedicado al desarrollo de las competiciones deportivas.

Estas diferencias entre chicos y chicas también se extrapolan según nuestra investigación al entorno educativo y es, en las clases de EF, donde los alumnos perciben que realizan una mayor actividad que las alumnas. En relación con estas diferencias, Beltrán, Devís, Peiró, y Brown (2012) sugieren que la EF sigue teniendo una orientación masculina, y que esto genera en las chicas menos posibilidades de participación y un sentimiento de desigualdad. En esta misma línea, Sánchez, Martos y López (2017) y Piedra, Pérez, Rebollo y Ries (2011), hablan sobre el modelo de enseñanza sexista en relación al tratamiento de género en muchos aspectos de la práctica de AF. Así, en el aula de EF, sus programaciones o contenidos derivan en una oferta de actividades con patrones androcéntricos, donde la oferta de juegos y deportes ofrece experiencias menos satisfactorias para las chicas, percibiéndose, de esta forma, una diferencia de género tanto por parte de los docentes como de los mismos alumnos.

En el estudio de Blández, Fernández y Sierra (2007), los autores señalaron que siguen existiendo estereotipos dentro del aula de EF, donde los estudiantes percibían una menor exigencia en las cualidades físicas o en el nivel de actividad. Por ello, las diferencias encontradas en nuestro estudio pueden estar relacionadas con las expectativas que mantenga el profesorado sobre el alumnado de diferentes géneros. En este sentido, Murillo, Julián, García, Abarca, y Zaragoza (2013) apuntaron que, según el tipo de actividad planteada en las clases de EF, los índices de participación de las chicas variaban, concluyendo que, para una mayor participación de estas, sería conveniente promover actividades no competitivas ni estereotipadas socialmente.

Las diferencias encontradas entre la percepción del alumnado y la de la figura docente en relación con la AF realizada, pueden explicarse en concordancia a los resultados obtenidos en la investigación de Zaragoza y Ortiz (2012), donde se apreciaron valores sobrestimados de AF por parte del alumnado, ya que los dos géneros consideraban ser menos sedentarios y más activos de lo que eran realmente. Es reseñable analizar las diferencias encontradas en este sentido, ya que la percepción de los maestros hacía la AF en 
ambos géneros son dispares debido a la sobrestimación de AF en los chicos y la subestimación en las chicas. Siguiendo con lo expuesto, los resultados mostraron que los maestros sobreestimaron la actividad de los alumnos y subestimaron la de las alumnas en diferentes momentos del día/semana. Para comprender esta situación, serían necesarios más estudios con el fin de profundizar en su análisis y entender los factores influyentes en estas percepciones.

Continuando con el entorno escolar, se hallaron importantes diferencias en momentos puntuales del día, especialmente, en las clases de EF y en los recreos escolares. Estas diferencias coinciden con estudios como los de Beighle, Morgan, Le Masurier y Pangrazi (2006) en los que se concluyó que los niños solían realizar actividades más activas, como jugar a diferentes deportes, y las niñas realizaban actividades de menos intensidad, como caminar o jugar a la comba. Según lo expresado, Escalante, Backx, Saavedra, García y Domínguez (2011) y Martínez, Aznar y Contreras (2015), apuestan por aprovechar dichos recreos como medio de fomento de la práctica física, promoviendo actividades de recreo que impliquen la participación activa del alumnado para generar una mayor motivación a la realización de acciones motrices. En el estudio de Cantó y Ruiz (2005), se determinó que el espacio utilizado para la realización de actividades en el patio escolar durante el tiempo de recreo, era mayor en el caso de los chicos que de las chicas, pudiendo influir en el nivel de AF de estas y concluyendo que existe una mala gestión del espacio según el género.

Esta desigualdad es percibida también por los maestros tal y como apuntan Castillo, Córdoba y Gil (2018) en su estudio, respaldando las nuevas tendencias coeducativas que intentan disipar los estereotipos durante el tiempo de recreo, abogando al recreo escolar como un espacio que brinda la oportunidad de fomentar las relaciones interpersonales entre los grupos escolares, reduciendo los conflictos y equiparando la ocupación del espacio entre chicos y chicas. Además, los resultados obtenidos en el estudio, muestran que tanto alumnos como maestros percibieron que el mayor tiempo de AF realizado diariamente se producía en el aula de EF, mostrando así la incidencia en el nivel de AF que esta materia genera en el alumnado. En consecuencia, en estudios como los de Martínez, Contreras, Aznar y Lera (2012), se concluyó que el 12,63\% de la AF realizada semanalmente por los estudiantes era realizada durante las sesiones de EF.

\section{Conclusión}

Se hace necesario conocer el nivel de AF de los jóvenes en función del género, ya que el estudio demuestra que existen diferentes percepciones entre el alumnado y los docentes, y entre chicos y chicas, hecho que dificulta la intervención igualitaria e influyente de los profesores de EF sobre la promoción de la AF.

\section{Referencias}

Ara, I., Vicente, G., Perez, J., Jimenez, J., Serrano, J.A., Dorado, C. \& Calbet, J.A. L. (2006). Influence of extracurricular sport activities on body composition and physical fitness in boys: a 3-year longitudinal study. International journal of obesity, 30(7), 1062. Recuperado de https:// www.nature.com/articles/0803303.

Arriscado, D., Muros, J., Zabala, M. \& Dalmau, J. (2015). ¿Influye la promoción de la salud escolar en los hábitos de los alumnos? Anales de pediatría, 83(1), 11-18. Recuperado de https://www.sciencedirect.com/science/article/ pii/S1695403314003968.

Beighle, A., Morgan, C. F., Le Masurier, G. \& Pangrazi, R. P. (2006). Children's physical activity during recess and outside of school. Journal of School Health, 76(10), 516520. Recuperado de https://onlinelibrary.wiley.com/doi/ abs/10.1111/j.1746-1561.2006.00151.x.

Beltrán, V. J., Devís, J., Peiró, C. \& Brown, D. H. (2012). When physical activity participation promotes inactivity: Negative experiences of Spanish adolescents in physical education and sport. Youth \& Society, 44(1), 3-27. Recuperado de https://journals.sagepub.com/doi/abs/10.1177/ $0044118 \times 10388262$.

Beltrán, V. J., Sierra, A. C., Jiménez, A., González, D., Martínez, C. \& Cervelló, E. (2017). Diferencias según género en el tiempo empleado por adolescentes en actividad sedentaria y actividad física en diferentes segmentos horarios del día. Retos. Nuevas Tendencias en Educación Física, Deporte y Recreación, 31. Recuperado de https:// www.redalyc.org/html/3457/345750049001/.

Blández, J., Fernández, E. \& Sierra, M. Á. (2007). Estereotipos de género, actividad física y escuela: La perspectiva del alumnado. Profesorado. Revista de Currículum y Formación de Profesorado, 11(2). Recuperado de https:/ /www.redalyc.org/pdf/567/56711206.pdf.

Brymer, E. \& Davids, K. (2016). Designing environments to enhance physical and psychological benefits of physical activity: a multidisciplinary perspective. Sports Medicine, 46(7), 925-926. Recuperado de https:// link.springer.com/article/10.1007/s40279-016-0535-8.

Cabrero, M, Aparicio, A., Pastor, T., Salinero, J., González, B., Fernández, J., ... Moreno, R. (2015). Actividad física y ocio y su relación con el índice de Ruffier en adolescentes. Revista Internacional de Medicina y Ciencias de la Actividad Física y del Deporte, 15(57), 165180. Recuperado de https://www.redalyc.org/pdf/542/ 54238756011.pdf.

Cantó, R. \& Ruiz, L. M. (2005). Comportamiento Motor Espontáneo en el Patio de Recreo Escolar: Análisis de las diferencias por género en la ocupación del espacio durante el recreo escolar. Revista Internacional de Ciencias del Deporte, 1(1), 28-45. Recuperado de https:// dialnet.unirioja.es/servlet/ articulo?codigo=1390035.

Castillo, G, Córdoba, C. C. P. \& Gil, P.(2018). Dinamización del recreo como resolución de conflictos y participación en actividades físico-deportivas. Revista Electrónica Educare, 22(2), 1-22. Recuperado de http://oaji.net/ articles/ 2017/2279-1525968969.pdf.

Delgado, M. \& Tercedor, P. (2002). Estrategias de intervención en educación para la salud desde la educación física. Barcelona: Inde.

Escalante, Y., Backx, K., Saavedra, J. M., García, A., \& Domínguez, A. M. (2011). Relación entre actividad física diaria, actividad física en el patio escolar, edad y sexo en escolares de educación primaria. Revista Española de 
Salud Pública, 85(5), 481-489. Recuperado de http:// scielo.isciii.es/pdf/resp/v85n5/07_original_breve1.pdf.

Estudio ALADINO (2015). Estudio de Vigilancia del Crecimiento, Alimentación, Actividad Física, Desarrollo Infantil y Obesidad en España 2015. Madrid: Agencia Española de Consumo, Seguridad Alimentaria y Nutrición. Ministerio de Sanidad, Servicios Sociales e Igualdad.

Gil, P., Perona, J. M., Prieto, A. \& Sáez, M. B. (2017). Evolución de los intereses y opiniones curriculares del área de educación física de padres y alumnos. Movimento, 23(3). Recuperado de https://www.redalyc.org/html/1153/ 115352985021/.

Iannotti, R. J., Janssen, I., Haug, E., Kololo, H., Annaheim, B. \& Borraccino, A. (2009). Interrelationships of adolescent physical activity, screen-based sedentary behaviour, and social and psychological health. International journal of public health, 54(2), 191-198. Recuperado de https:// link.springer.com/article/ 10.1007/s00038-009-5410-z.

Kalman, M., Inchley, J., Sigmundova, D., Iannotti, R. J., Tynjälä, J. A., Hamrik, Z., ... Bucksch, J. (2015). Secular trends in moderate-to-vigorous physical activity in 32 countries from 2002 to 2010: a cross-national perspective. The European Journal of Public Health, 25(2), 37-40. Recuperado de https://academic.oup.com/eurpub/articleabstract/25/suppl_2/37/589675.

Lavielle, P., Pineda, V., Jáuregui, O. \& Castillo, M. (2014). Actividad física y sedentarismo: Determinantes sociodemográficos, familiares y su impacto en la salud del adolescente. Revista de salud pública, 16, 161-172. Recuperado de https://www.scielosp.org/ scielo.php?pid=S0124-00642014000200001\&script= sci_arttext\&tlng=pt.

Manchola, J., Bagur, C. \& Girabent, M. (2017). Fiabilidad de la versión española del cuestionario de actividad física PAQ-C. Revista Internacional de Medicina y Ciencias de la Actividad Física y del Deporte, 17(65). Recuperado de https://dialnet.unirioja.es/servlet/articulo?codigo= 5896428.

Martínez, D., Martínez, V., Pozo, T., Welk, G. J., Villagra, A., Calle, M. E., Marcos, A. \& Veiga, O. L. (2009). Reliability and validity of the PAQ-A questionnaire to assess physical activity in Spanish adolescents. Revista española de salud pública, 83(3), 427-439. Recuperado de https://europepmc.org/abstract/med/ 19701574.

Martínez, J., Aznar, S. \& Contreras, O. (2015). El recreo escolar como oportunidad de espacio y tiempo saludable. Revista Internacional de Medicina y Ciencias de la Actividad Física y del Deporte, 59, 419-432. Recuperado de https://revistas.uam.es/rimcafd/article/view/ rimcafd2015.59.002.

Martínez, J., Contreras, O. R, Aznar, S. \& Lera, Á. (2012). Niveles de actividad física medido con acelerómetro en alumnos de $3^{\circ}$ ciclo de educación primaria: actividad física diaria y sesiones de educación física. Revista de Psicología del Deporte, 21(1), 117-123. Recuperado de http:/ /www.rpd-online.com/article/view/939.

Murillo, B., Julián, J. A., García, L., Abarca, A. \& Zaragoza, J. (2013). Influencia del género y de los contenidos sobre la actividad física y la percepción de competencia en Educación Física. Revista Internacional de Ciencias del
Deporte, 10(36), 131-143. Recuperado de https:// www.cafyd.com/REVISTA/ ojs/index.php/ricyde/article/ view/621.

Organización Mundial de la Salud (2010). Recomendaciones mundiales sobre actividad física para la salud. World Health Organization: Ginebra, Suiza 2010.

Organización Mundial de la Salud(OMS) (2017). Obesidady sobrepeso. Recuperado de http://www.who.int/ mediacentre/factsheets/fs311/es/.

Oyarzún, J. C., Wydra, G., Hernández, C. \& Salazar, C. M. (2017). Actitudes hacia la educación física: Grados de importancia y conformidad según escolares de Chile y Alemania. Una mirada intercultural. Retos: nuevas tendencias en educación física, deporte y recreación, 32, 158-162. Recuperado de https://dialnet.unirioja.es/ servlet/articulo?codigo=6352294.

Piedra, J., Pérez, R. G., Rebollo, Á. \& Ries, F. (2011). Actitudes hacia la coeducación en Andalucía ¿es el profesorado de Educación Física diferente? Revista de investigación en educación, 2(9), 200-208. Recuperado de https:// dialnet.unirioja.es/ servlet/articulo?codigo $=4731574$.

Pinel, C., Chacón, R., Castro, M., Espejo, T., Zurita, F., \& Pérez, A. (2017). Diferencias de género en relación con el Índice de Masa Corporal, calidad de la dieta y actividades sedentarias en niños de 10 a 12 años. Retos: nuevas tendencias en educación física, deporte y recreación, (31), 176-180. Recuperado de https://recyt.fecyt.es/ index.php/retos/article/view/49393/32170.

Ramos, P., Rivera, F. J., Jiménez, A. M. \& Moreno, M. D. C. (2012). Análisis de clúster de la actividad física y las conductas sedentarias de los adolescentes españoles, correlación con la salud biopsicosocial. Revista de Psicología Del Deporte, 21(1), 99-106. Recuperado de https:/ /idus.us.es/xmlui/bitstream/handle/11441/23184/ file_1.pdf?sequence $=1$.

Reverter, J., Plaza, D., Jové, M. C., \& Hernández, V. (2014). Actividad físico-deportiva extraescolar en alumnos de primaria: el caso de Torrevieja (Alicante). Retos. Nuevas tendencias en Educación Física, Deporte y Recreación, 2014, núm. 25, p. 48-52. Recuperado de https:// www.redalyc.org/html/3457/345732291010/.

Sánchez, N., Martos, D. \& López, A. (2017). Las mujeres en los materiales curriculares: el caso de dos libros de texto de educación física. Retos: nuevas tendencias en educación física, deporte y recreación, (32), 140-145. Recuperado de https://www.redalyc.org/html/3457/345751100028/

Williams, E. P., Mesidor, M., Winters, K., Dubbert, P. M. \& Wyatt, S. B. (2015). Overweight and obesity: prevalence, consequences, and causes of a growing public health problem. Current obesity reports, 4(3), 363-370. Recuperado de https://link.springer.com/article/10.1007/ s13679-015-0169-4.

Zaragoza, A., \& Ortiz, R. (2012). Estado nutricional y nivel de actividad física según percepción de los estudiantes de la Universidad de Alicante. Revista Española Nutrición Comunitaria, 18(3), 149-153. Recuperado de http:// rua.ua.es/dspace/handle/10045/31984. 